\title{
Role of Disorder on the Quantum Critical Point of a Model for Heavy Fermions
}

\author{
T. G. Rappoport, A. Saguia, B. Boechat and M. A. Continentino \\ Departamento de Física - Universidade Federal Fluminense \\ 24210-130, Niterói , RJ - Brazil; e-mail: amen@if.uff.br
}

(October 31, 2018)

\begin{abstract}
A zero temperature real space renormalization group $(\mathrm{RG})$ approach is used to investigate the role of disorder near the quantum critical point (QCP) of a Kondo necklace (XY-KN) model. In the pure case this approach yields $J_{c}=0$ implying that any coupling $J \neq 0$ between the local moments and the conduction electrons leads to a non-magnetic phase. We also consider an anisotropic version of the model $(X-K N)$, for which there is a quantum phase transition at a finite value of the ratio between the coupling and the bandwidth, $(J / W)$. Disorder is introduced either in the onsite interactions or in the hopping terms. We find that in both cases randomness is irrelevant in the $X-K N$ model, i.e., the disorder induced magnetic-non-magnetic quantum phase transition is controlled by the same exponents of the pure case. Finally, we show the fixed point distributions $P_{J}(J / W)$ at the atractors of the disordered, non-magnetic phases.
\end{abstract}

PACS :75.10.Hk; 64.60.Ak; 64.60.Cn

Heavy-fermion materials exhibit a variety of interesting phenomena and different ground states including antiferromagnetic, superconductor, Kondo-insulator and metallic [1]:2]. Most of the properties of these systems can be attributed to their proximity to a magnetic quantum critical point $(Q C P)$ [3]. An important and actual problem in the study of these materials is to understand the role of disorder on the quantum critical point. This is also a general problem in statistical mechanics which had a lot of progress in the last years mainly due to the study of random quantum spin chains [4]. For heavy fermions it is particularly relevant to understand the role of disorder since a standard way of bringing these systems to the $Q C P$ is by doping, which necessarily perturbs the system [5].

The disordered Kondo model [6] and the Griffiths phase approach [7] have been proposed to deal with disorder in heavy fermions. In spite of bringing progress to this field they are, at this point, mostly phenomenological theories. It is important that results can be obtained starting from a microscopic Hamiltonian which is closest to describe heavy fermions and that can incorporate disorder at a basic level. In this Communication we present a non-perturbative real space renormalization group approach to the one-dimensional Kondo necklace $(K N)$ model at zero temperature, both with and without disorder. This model was proposed by Doniach [8] to study heavy fermions and emphasizes magnetic degrees of freedom [9 12 neglecting charge fluctuations. It incorporates the essential physics of these systems, namely the competition between Kondo effect and magnetic ordering. The quantum phase transition of this model is a magnetic transition which is the actual case in most heavy fermions materials. As far as we know the role of disorder in the $K N$ model has never been considered and the present Communication represents a first step towards describing its effects in heavy fermions starting at a microscopic level. The $K N$ model is given by the Hamiltonian,

$$
H=\sum_{i=1}^{L-1} W_{i}\left(\sigma^{x}{ }_{i} \sigma^{x}{ }_{i+1}+\sigma^{y}{ }_{i} \sigma^{y}{ }_{i+1}\right)+\sum_{i=1}^{L-1} J_{i} \vec{S}_{i} \cdot \vec{\sigma}_{i},
$$

where $\sigma^{\mu}$ and $S^{\mu}, \mu=x, y, z$ are spin- $1 / 2$ Pauli matrices denoting the spin of the conduction electrons and those of the local moments, respectively. The sites $i$ and $i+1$ are nearest-neighbors on a chain of $L$ sites and $W_{i}$ is an antiferromagnetic coupling which represents the hopping of the conduction electrons between neighboring sites. In the present study, either the on-site interactions between the conduction electrons and the local moments, $J_{i}>0$ or the hopping $W_{i}$ are uncorrelated quenched random variables with initial probability distributions, $P_{J}\left(J_{i} / W\right)$ and $P_{W}\left(J / W_{i}\right)$ respectively. A closely related model, which we also study here is the $X$-Kondo necklace $(X-$ $K N)$ model where the band of conduction electrons is represented just by the Ising term, $\sum_{i=1}^{L-1} W_{i} \sigma^{x}{ }_{i} \sigma^{x}{ }_{i+1}$.

In the pure case our zero temperature RG calculations yield distinct results for these models. For the $X-K N$ we find an unstable fixed point at a finite value of $(J / W)$ separating an antiferromagnetic phase from a spin compensated, Kondo-like phase, which can be fully characterized. For the $X Y-K N$, any interaction $J$ gives rise to a dense Kondo state. The latter result, $J_{c}=0$, for the $X Y-K N$ is in agreement with the most recent densitymatrix renormalization group 13 and quantum Monte Carlo calculations [14].

The introduction of disorder in the on-site interactions $J$ or in the hopping terms leads us to consider the renormalization of the probability distributions, $P_{J}(J / W)$ and $P_{W}(J / W)$. For the $X-K N$ model, disorder either in the interactions or in the hopping turns out to be an irrelevant perturbation at the pure system fixed point, i.e., the zero temperature exponents associated with the transition from the antiferromagnetic $(A F)$ phase to the nonmagnetic phase in the presence of disorder are those of the pure case. This is in strong contrast with the Ising model in a transverse field, which has been used as a model for heavy fermions [7], for which disorder is a rel- 
evant perturbation at $d=1$ [15] and probably also for $d<4$ [16]. The atractor of the antiferromagnetic phase in the presence of disorder is the same of the pure case. There is a new atractor associated with the disordered non-magnetic phase which is characterized by the fixed point probability distributions $P_{J}^{*}(J / W)$ and $P_{W}^{*}(J / W)$ at this atractor in each case. In the $X Y-K N$ model the flows are always towards non-magnetic phases, which are different in the pure and disordered cases. The fixed point distributions $P_{J}^{*}(J / W)$ in the non-magnetic phases of the $X-K N$ and the $X Y-K N$ in the presence of disorder are extremely wide. They show huge values of $(J / W)$ and negligeable weight for small values of the ratio $(J / W)$ which are progressively being eliminated.

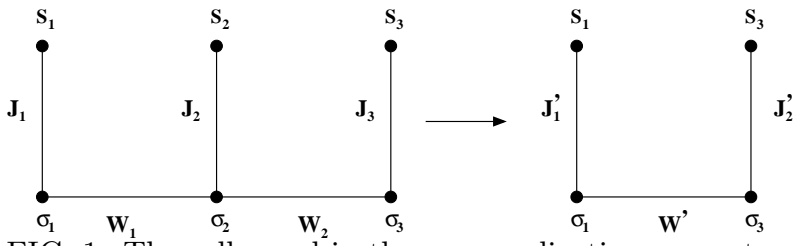

FIG. 1. The cells used in the renormalization group transformation and which correspond to a length scale factor $b=2$.

In the context of the real-space renormalization group, the linear chain may be viewed as $b$ bonds in series; $b$ is the scaling factor $(b=2$ in Fig. 1). For the Hamiltonian given in Eq. 1, the non-commutation aspects are present at the cluster level, in the sense that individual spins are not in a definite state. This can be dealt with by referring to the density matrix in the basis $\left|m_{1} m_{2} \ldots m_{n} m_{1}^{\prime} m_{2}^{\prime} \ldots m_{n}^{\prime}\right\rangle$, where $S^{x}\left|m_{i}^{\prime}\right\rangle=m_{i}^{\prime}\left|m_{i}^{\prime}\right\rangle$ and $\sigma^{x}\left|m_{i}\right\rangle=m_{i}\left|m_{i}\right\rangle$, and defining the renormalizationgroup ( $\mathrm{RG}$ ) transformation by the mapping of diagonal elements only. At zero temperature the density matrix is essentially the ground state projector. This approach has been successfully applied to the transverse Ising model [17 and to quantum spin glasses [18]. The disorder is incorporated through a statistical renormalization group (SRG) treatment where one follows the effect of a RG transformation on the probability distributions of the relevant parameters, instead of forcing them to a particular form 18]. Let us consider the case of random interactions $J$. For a given configuration of onsite interactions $\left(\left\{J_{i} / W\right\}\right)$ the $\mathrm{RG}$ transformation for the $d=1$ system (Fig. ) is defined by,

$$
\left\langle m_{1} m_{3}\left|\tilde{\rho}^{\prime}\left(K^{\prime}\right)\right| m_{1} m_{3}\right\rangle=\left\langle m_{1} m_{3}\left|\tilde{\rho}\left(K_{1}, K_{2}, K_{3}\right)\right| m_{1} m_{3}\right\rangle
$$

where $K^{\prime}=(J / W)^{\prime}$ is the renormalized ratio of parameters in the smallest two-site cell. We have assigned a single index $i$ to each bond in the original cell $K_{i}=J_{i} / W$ consistent with an uncorrelated distribution for these ratios. The matrix elements,

$$
\left\langle m_{1} m_{3}\left|\tilde{\rho}^{\prime}\left(K^{\prime}\right)\right| m_{1} m_{3}\right\rangle=
$$

$$
\sum_{m_{1}^{\prime}, m_{3}^{\prime}}\left\langle m_{1} m_{3} m_{1}^{\prime} m_{3}^{\prime}\left|\tilde{\rho}^{\prime}\left(K^{\prime}\right)\right| m_{1} m_{3} m_{1}^{\prime} m_{3}^{\prime}\right\rangle
$$

and

$$
\begin{gathered}
\left\langle m_{1} m_{3}\left|\tilde{\rho}\left(K_{1}, K_{2}, K_{3}\right)\right| m_{1} m_{3}\right\rangle= \\
\sum_{m_{2}, m^{\prime}{ }_{1}, m^{\prime}{ }_{2}, m^{\prime}{ }_{3}}\left\langle m_{1} m_{2} m_{3} m^{\prime}{ }_{1} m^{\prime}{ }_{2} m^{\prime}{ }_{3}\right| \\
\rho\left(K_{1}, K_{2}, K_{3}\right)\left|m_{1} m_{2} m_{3} m^{\prime}{ }_{1} m^{\prime}{ }_{2} m^{\prime}{ }_{3}\right\rangle
\end{gathered}
$$

are obtained by performing the partial trace on the internal spins keeping those on the terminal sites (along the chain) fixed (see Fig.). For the renormalized cell, the left hand side of Eq (2) provides analytical expressions for the primed variables which are given by,

$$
\left\langle++\left|\tilde{\rho}^{\prime}\left(K^{\prime}\right)\right|++\right\rangle=-\frac{1}{4}\left[\frac{1-\sqrt{1+16 K^{\prime 2}}}{\sqrt{1+16 K^{\prime 2}}}\right]
$$

and

$$
\left\langle-+\left|\tilde{\rho}^{\prime}\left(K^{\prime}\right)\right|+-\right\rangle=\frac{1}{4}\left[\frac{1+\sqrt{1+16 K^{\prime 2}}}{\sqrt{1+16 K^{\prime 2}}}\right]
$$

For the original, larger cell, the right hand side of Eq. (2) is written in a 64 by 64 representation and the calculations have to be performed numerically. The matching of these quantities above in the two cells yields in principle two RG equations that should allow to treat $J_{i}$ and $W_{i}$ as independent random variables. It results however that these equations reduce to a single one which points out that, as concerns the ground state of the system, there is a unique relevant variable in the problem, namely, the ratio $(J / W)$. This is clear in the pure system but turns out to be the case also in the presence of disorder as is further discussed below

For convenience let us define,

$$
Z_{ \pm}=\langle++|\tilde{\rho}|++\rangle \pm\langle-+|\tilde{\rho}|+-\rangle
$$

for both $b=1$ and $b=2$ cells. We then match the quantity $Z_{-}^{\prime} / Z_{+}^{\prime}=Z_{-} / Z_{+}$, which is a scale invariant and finally obtain,

$$
K^{\prime}=\frac{1}{4} \sqrt{\left(\frac{Z_{+}}{Z_{-}}\right)^{2}-1}
$$

which is our basic renormalization group equation. We emphasize that although we have considered different scale invariants to match in the distinct cells we always find the same RG equation or trivial ones 19. We obtain below that the cases of disorder in the interactions or in the hopping yield the same phase diagram. These results are consistent with the existence of a single random variable in the problem, the ratio $(J / W)$, that suffices to characterize the ground state phase diagram. Furthermore, as we take $K_{i}=J_{i} / W$ or $G_{i}=J / W_{i}$ as random 
variables, $W$ or $J$ respectively, are uniform only at the level of the cells and not in the chain generated by the RG procedure.

We first consider the pure case without disorder. For the $X-K N$ these equations yield a quantum critical point at $K_{c}=G_{c}=(J / W)_{c}=0.21$ separating an antiferromagnetic from a non-magnetic, Kondo singlet or spin-liquid phase. The critical exponents associated with this transition can be obtained in the usual way. For the correlation length, given by $\xi \propto|\delta|^{-\nu}$, with $\delta=K-K_{c}$, we obtain the exponent, $\nu=2.24 \approx 9 / 4$. The dynamic exponent $z$ is obtained from the finite size scaling relation for the gaps for excitations in the two cells, i.e., $\left(\Delta E / \Delta E^{\prime}\right)_{K_{c}}=\left(L^{\prime} / L\right)^{z}=b^{z}$, at the quantum critical point, where $b=2$ is the length scale factor. We get $z=1.28$. Using the quantum hyperscaling relation $2-\alpha=\nu(d+z)$ we can also obtain the exponent $\alpha=-3.12$ which is negative but large in module. The non-magnetic phase, for $K>K_{c}$, is a spin-liquid phase characterized by a gap $\Delta E=|\delta|^{\nu z}$ which vanishes at the QCP with the gap exponent, $s=\nu z=2.28$. For the $X Y-K N$ model we find that any finite coupling $J$ gives rise to a non-magnetic, Kondo-like phase, i.e., $K_{c}=0$.

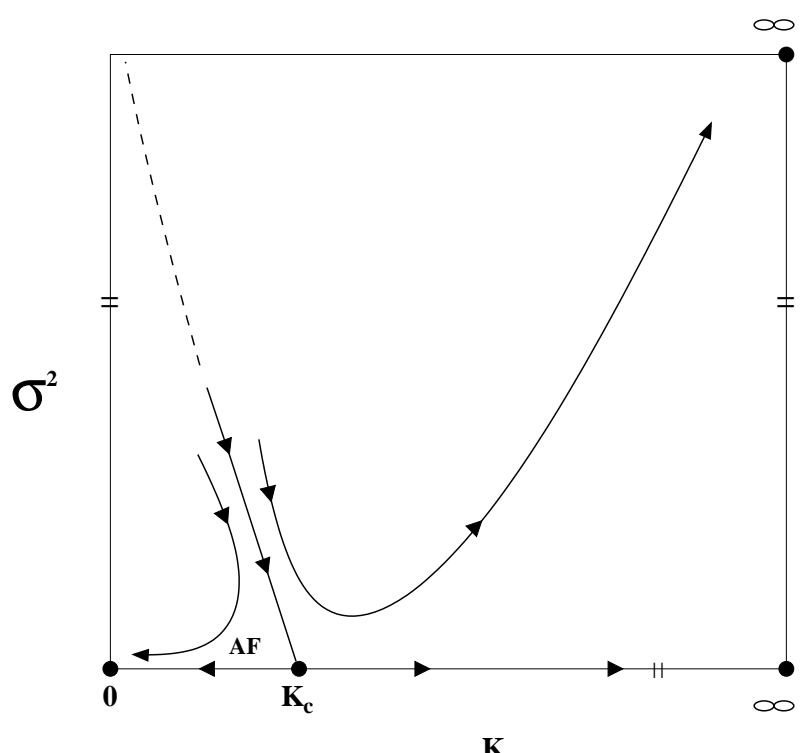

FIG. 2. Schematic phase diagram of the $X-K N$ model obtained from our renormalization group method. The arrows give the flow of the $\mathrm{RG}$ equations. The fixed point at $K_{c}=(J / W)_{c}, \sigma^{2}=0$ is that of the pure system.

The effects of disorder in the on-site interactions in the pure Kondo necklace model, are considered taking, for convenience, a normalized initial probability distribution, $P\left(K_{i}\right)=\left(K_{i}^{\alpha-1} / \beta^{\alpha} \Gamma(\alpha)\right) \exp \left(-K_{i} / \beta\right)$. This is a single peaked distribution with an average value $\langle K\rangle=\alpha \beta$ and a variance $\sigma^{2}=\left\langle(K-\langle K\rangle)^{2}\right\rangle=\alpha \beta^{2}$. Notice that $K \geq 0$, i.e., only positive (antiferromagnetic) values of the on-site interactions are considered. We start the iteration of the RG equation, choosing three bonds distributed according to $P\left(K_{i}\right)$ above, to feed the recursion relations and generate a new value $K^{\prime}$. This procedure is repeated up to $10^{6}$ times and yields a new renormalized distribution $P^{\prime}\left(K^{\prime}\right)$ which will be used to feedback the recursion relation in the next step of the renormalization process. The evolution of the distribution is then followed as the renormalization process goes on. We emphasize that the exact form of the initial probability distribution is not relevant and the different starting distributions we used always evolve to the same fixed point form associated with the atractor of the corresponding phase.

We next consider adding randomness to the on-site interactions of the pure $X-K N$ model. The zerotemperature RG flow diagram as a function of $\langle K\rangle=K$ and $\sigma^{2}$ is shown in Fig. 2. In the presence of disorder the system still exhibits two phases: the Ising antiferromagnetic phase $(X-A F)$ and a disordered, non-magnetic phase. The atractor of the antiferromagnetic, $X-A F$, phase in the presence of disorder is the same of the pure $A F$ phase. The probability distribution in this ordered phase is single peaked and becomes sharper, i.e., $\sigma^{2} \rightarrow 0$ as the mean value approaches the value $\langle K\rangle=0$ under successive renormalizations (see Fig.3). On the other hand, it is clear from the phase diagram in Fig. 2 that the atractor of the non-magnetic phase in the presence of disorder is different from the atractor of the non-magnetic, spin-liquid phase of the pure case.

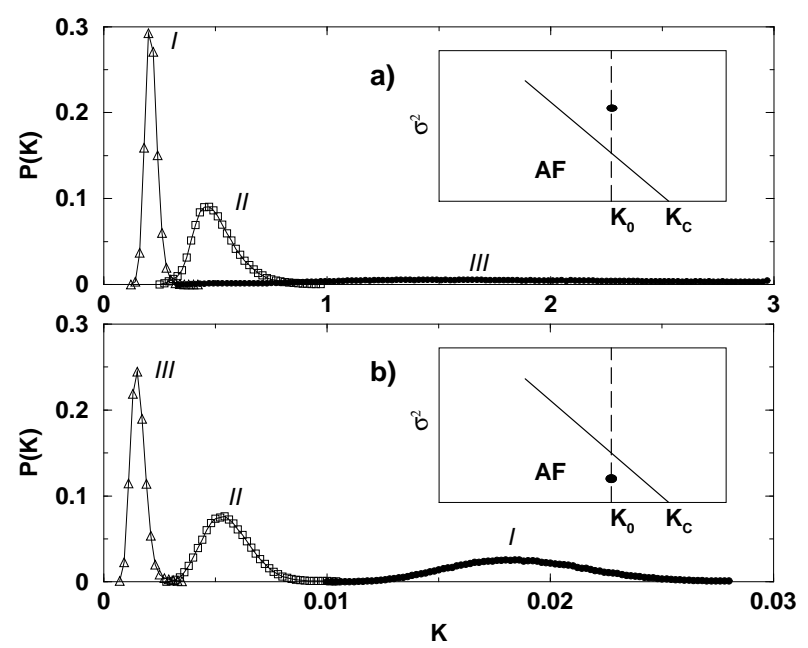

FIG. 3. The probability distributions $P_{J}(J / W)$ at two sucessive steps of the statistical renormalization procedure $(I$ and $I I$ respectively) as the system iterates to the atractor of the disordered, non-magnetic phase $(a)$ and to the atractor of the antiferromagnetic phase $(b)$ of the $X-K N$ model. The insets show the initial points of the RG processes. The critical boundary in Fig. separates the two types of flow shown here.

The phase transition to the ordered magnetic phase in the presence of disorder is governed by the same critical exponents of the pure case that we have calculated before. This is a consequence that the flow along the critical line is towards the semi-stable fixed point of the pure system at $K_{c}=0.21, \sigma=0$ which therefore determines the universality class of the transition, even in the presence of 
disorder. The irrelevance of disorder in the renormalization group sense is consistent, according to Harris criterion, with the negative value of the exponent $\alpha=-3.12$ we have obtained previously. The critical line close to the QCP vanishes as, $\sigma_{c}^{2} \propto|\delta|=\left|K-K_{c}\right|$. The exact form of the critical frontier in the region represented by the dashed line is not numerically accessible. Most probably it approaches asymptotically the axis $K=0$ as there should be no critical value for this type of disorder to destroy the $X-A F$ phase at $K=0$. Notice that disorder is detrimental to antiferromagnetism, reducing the region of the phase diagram where this can be found. We have also carried out the statistical RG procedure for the case of disorder in the hopping terms using the same initial distributions, but now for the random variable $G_{i}=J / W_{i}$. It turns out that the same phase diagram of Fig.2 is obtained.

The effects of disorder in the on-site interactions on the $X Y-K N$, which in the pure case has only a nonmagnetic Kondo phase, are quite distinct, from the RG point of view, compared with those in the anisotropic $X-$ $K N$ model. Firstly, disorder is a relevant perturbation at the $K_{c}=0$ fixed point since for any initial values of $\langle K\rangle \neq 0$ and $\sigma^{2}$, the renormalized variance always increases in the renormalization process. Next, the flow of the distribution $P(J / W)$ towards the atractor of the random Kondo phase is much faster than for the $X-$ $K N$. The phase diagram of the $X Y-K N$ has only two atractors, that of the pure Kondo phase at $\sigma=0$, $J / W=\infty$ and that of the random Kondo phase at $\sigma=$ $\infty,\langle K\rangle=\infty$. This is the first time this result has been found using a real space RG. The same considerations apply for the case of off-diagonal disorder, i.e., when the hoppings are random, with the replacement $\langle K\rangle \rightarrow\langle G\rangle$.

In the non-magnetic phases, of both the disordered $X-K N$ and $X Y-K N$ models, for the case of disorder in the interactions $J_{i}$, the distributions $P_{J}(K)$ become extremely wide under iteration as shown in Fig. 3. Small values of $K$ are being eliminated and huge values for this ratio are being generated. Note that this behavior of $P_{J}(K)$ does not imply the absence of low energy excitations since large values of $K$ can occur for $J$ finite and $W \rightarrow 0$ [11]. In fact the only meaningful variable in the present approach is the ratio of these quantities. These disordered phases are better characterized by the distribution of gaps for excitations which however can not be obtained in the present approach since it does not take into account excited states.

In summary, we have studied the effect of disorder in the $K N$ model for heavy fermion systems. The anisotropic version of the model, the $X-K N$, is appropriate to describe systems where the ordered magnetic phase has a strong Ising character. In this case for pure systems we have found a quantum critical point, at a finite value of $(J / W)$, separating the ordered magnetic phase from the spin-liquid. Remarkably, we have obtained that at $d=1$, where the effects of disorder are stronger, this turns out to be an irrelevant perturbation at the pure system quantum critical point. This holds for both cases of initial disorder in the interactions $J_{i}$ or in the hoppings $W_{i}$. The method used here has the great advantage of being non-perturbative which allows to the treat the effects of weak disorder in the neighborhood of the quantum critical point of the pure system. Our results are in agreement with the robustness of the quantum critical point approach description of nonFermi liquid behavior in systems where disorder is clearly present. The present RG method characterizes the different phases in terms of their atractors. In particular, in the disordered phases the same atractor is reached independently of the point of the phase diagram we start the statistical iteration procedure. Consequently the present approach does not yield information on the existence of rare clusters of the incipient magnetically ordered phase which characterizes the so-called Griffiths phases. This is a feature of the method which considers only the ground state of the cells and no excited states are involved in the calculations. Another consequence of this feature is the existence of a single variable, the ratio $(J / W)$ which suffices to characterize the phase diagram of the system even in the presence of disorder. This approach however can be extended to include excited states and to calculate the distribution of gaps in which case we expect to find evidence of Griffiths phases 21].

\section{ACKNOWLEDGMENTS}

The authors are grateful to the Brazilian agencies FAPERJ, CNPq and CAPES for financial supports.

[1] J.D. Thompson and J. M. Lawrence, in Handbook on the Physics and Chemistry of Rare Earths, Chapter 133, p. 383, Vol.19 - Lanthanides/Actinides:Physics-II, edited by K.A. Gschneider Jr., L. Eyring, G.H. Lander and G.R. Choppin, Elsevier Science B.V., 1994.

[2] G. Aeppli and Z. Fisk, Comments Condens. Matter. Phys. 16, 155 (1992).

[3] see M.A. Continentino, Quantum Scaling in Many-Body Systems (World Scientific, Singapore, 2001).

[4] S. K. Ma, C. Dasgupta, and C.-K. Hu, Phys. Rev. Lett. 43, 1434 (1979); D. S. Fisher, Phys. Rev. B 50, 3799 (1994); R. A. Hyman and K. Yang, Phys. Rev. Lett. 78, 1783 (1997); C. Monthus, O. Golinelli, and Th. Jolicoeur, Phys. Rev. Lett. 79, 3254 (1997); D.S. Fisher and A.P. Young, Phys. Rev. B 58, 9131 (1998).

[5] M.C. Aronson et al., Phys. Rev. Lett. 75, 725 (1995).

[6] E. Miranda, V. Dobrosavljevic and G. Kotliar, Phys. Rev. Lett. 78, 290 (1997).

[7] Chia-Ying Liu, et al., Phys. Rev. B61, 432 (2000); A.H. Castro Neto and B.A. Jones, Phys. Rev. B62, 14975 (2000). 
[8] S. Doniach, Physica 91 B, 231 (1977).

[9] R. M. Fye and D. J. Scalapino, Phys. Rev. B 44, 7486 (1991).

[10] H. Tsunetsugu, Y. Hatsugai, K. Ueda and M. Sigrist, Phys. Rev. B 46, 3175 (1992)

[11] R. Jullien, P. Pfeuty, A. K. Bhattacharjee and B. Coqblin, J. Appl. Phys. 50, 7555 (1979).

[12] C. C. Yu and S. R. White, Phys. Rev. Lett., 71, 3866 (1993).

[13] S. Moukouri, L. G. Caron, C. Bourbonnais and L. Hubert, Phys. Rev. B51, 15920 (1995).

[14] R. T. Scalettar, D. J. Scalapino. and R. J. Sugar, Phys. Rev. B 31, 7316 (1985).

[15] H. Rieger and A. P. Young, Phys. Rev. B54, 3328 (1995).

[16] O. Motrunich, S-C. Mau, D.A. Huse and D. Fisher, Phys. Rev. B 61, 1160 (2000).

[17] R. R. dos Santos, J. Phys. C 15, 3141 (1981).

[18] B. Boechat, R. R. dos Santos and M. A. Continentino, Phys. Rev. B 49, 6404 (1994); M. A. Continentino, B. Boechat and R. R. dos Santos, Phys. Rev. B 50, 13528 (1994).

[19] Z. Friedman, Phys. Rev. B17, 1429 (1978).

[20] M.A. Continentino, G. Japiassu and A. Troper, Phys. Rev. B, (1989); S. Sachdev and J.W. Ye, Phys. Rev. Lett. 69, 2411 (1992); M.A. Continentino, Phys. Rev. B47 , 11587 (1993); A. M. Tsvelik and M. Reizer, ibid. B48, 9887 (1993); A.J. Millis, ibid. B48, 7183 (1993); T. Moriya and T. Takimoto, J. Phys. Soc. Jpn. 64, 960 (1995); M.A.Continentino, Eur. Phys. Journal B13, 31 (2000).

[21] T.G. Rappoport, A. Saguia, B. Boechat and M.A. Continentino, to be published in Physica B. 